\section{Molecular and immuno- cytochemical identification of coxsackievirus A-24 variant from the acute haemorrhagic conjunctivitis outbreak in Taiwan in 2007}

\begin{abstract}
Aims Acute haemorrhagic conjunctivitis (AHC) is a highly contagious conjunctivitis associated with enteroviruses. Coxsackie A-24 variant (CA-24v) and enterovirus-70 (EV-70) are the two major causative agents. During October 2007, an epidemic of AHC occurred in Taiwan, affecting more than 11000 people. The aim of this study was to determine the aetiological agent associated with the outbreak in patients diagnosed with $\mathrm{AHC}$ and treated at the Cathay General Hospital, Taipei (CGHT) and Cathay General Hospital Sijhih (CGHS), Taiwan during October 2007.

Methods Virus isolates were obtained from six patients (four from CGHS and two from $\mathrm{CGH}$ ), and a total of seven specimens (one throat and one rectal, and five eye swabs) were collected. The specimens were inoculated onto the MRC- 5 cell lines. The viral isolation was confirmed by performing real-time reverse transcription-polymerase chain reaction (RT-PCR) and indirect immunofluorescence assay (IFA).

Results The conjunctival, throat, and rectal swabs collected in this study were all tested positive for a variant of CA-24. All seven viral isolates were characterized as a variant of CA-24 and confirmed by IFA and real-time RT-PCR.

Conclusions The findings suggest that the outbreak of AHC that occurred during October 2007 in the northern area of Taiwan was caused
\end{abstract}

P-C Kuo ${ }^{1,8}$, J-Y-S Lin ${ }^{2,3,8}$, L-C Chen ${ }^{4}$, Y-T Fang ${ }^{5}$,

Y-C Cheng ${ }^{5}, \mathrm{H}-\mathrm{Y} \mathrm{Wu}^{4}, \mathrm{C}-\mathrm{Y} \mathrm{Tsai}^{4}, \mathrm{Y}-\mathrm{S}$ Chen ${ }^{6}$,

S-Y Lin ${ }^{7}, C-L W u^{2}$ and Q-D Ling 3,5

Departments of Ophthalmology

and Medicine,

Cathay General Hospital

Sijhih Branch,

School of Medicine, Fu Jen

Catholic University, Taipei,

Taiwan, Republic of China

${ }^{2}$ Department of

Ophthalmology, Taipei

Medical University

Municipal Wan-Fang

Hospital, Taipei, Taiwan,

Republic of China

${ }^{3}$ Systems Biology and

Bioinformatics Institute,

by a variant of coxsackie A-24. Further phylogenic analysis is underway to further classify this CA-24v strain.

Eye (2010) 24, 131-136; doi:10.1038/eye.2009.8;

published online 13 February 2009

Keywords: acute haemorrhagic conjunctivitis; coxsackievirus A24 variant; indirect immunofluorescence assay; real-time reverse transcription-polymerase chain reaction

\section{Introduction}

Acute haemorrhagic conjunctivitis (AHC) is a highly contagious conjunctivitis characterized by a short incubation period, an explosive onset of signs and symptoms, bilateral ocular involvement, and a rapid recovery phase without significant ocular sequelae. Clinical presentations include lid oedema, watery discharge, conjunctival injection, follicular reaction, punctate epithelial keratitis, and typically subconjunctival haemorrhages. ${ }^{1}$ Systemic manifestations, such as upper respiratory symptom, cough, pharyngitis, myalgia, malaise, headache, gastrointestinal illness, fever, and periauricular lymphadenopathy, were also noted in some AHC patients. Symptoms usually start after an incubation period of 24-48 h, persist for 3-5 days and disappear within 1-2 weeks. Most cases are self-limited without any sequelae. ${ }^{2}$
National Central University, Chungli, Taiwan, Republic of China

${ }^{4}$ Department of Laboratory Medicine, Cathay General Hospital Sijhih Branch, Taiwan, Republic of China

${ }^{5}$ Cell Biology Laboratory, Cathay Medical Research Institute, Cathay General Hospital, Taipei, Taiwan, Republic of China

${ }^{6}$ Department of Ophthalmology, Cathay General Hospital Hsinchu Branch, Taiwan, Republic of China

${ }^{7}$ Department of Ophthalmology, Cathay General Hospital, Taipei, Taiwan, Republic of China

Correspondence: Q-D Ling, Cell Biology Laboratory, Cathay Medical Research Institute, Cathay General Hospital, Taipei, Taiwan, Republic of China Tel: + 886226907965 ext2313;

Fax: + 886226907963.

E-mail: qdling@cgh.org.tw ${ }^{8}$ These authors contributed equally to this work

Received: 12 June 2008 Accepted in revised form: 5 January 2009 Published online: 13 February 2009 
Adenovirus 11, coxsackie A-24 variant (CA-24v), and enterovirus-70 (EV-70) are the three major viruses known to cause AHC epidemics and pandemics. ${ }^{2,3}$ Compared with adenovirus 11, enteroviruses (EV-70 and CA-24v) are more likely to lead to the expanded epidemics because of their RNA genomes and short incubation period (6-12 $\mathrm{h}$ for the enterovirus compared with 2-6 days for the adenovirus). It is known that RNA viruses, such as enterovirus, may evolve at rates approximately $10^{6}$-fold faster than the adenovirus, which has the DNA genome. CA-24v is an antigenetic variant of CA-24, which is responsible for most occurrences of AHC outbreaks since 1970 in Singapore. ${ }^{1}$ There are three genotypes, designated as genotypes I-III, of the CA-24v that are identified by the phylogenetic analysis of the $3 \mathrm{C}$ protease region. Genotypes I and II correspond to strains isolated from Singapore, Hong Kong, and Thailand during the 1970s. Genotype III strains were isolated between 1985 and 1995 in Japan, Taiwan, China, Thailand, Singapore, and Ghana. The high degree of infectivity of CA-24v to the human conjunctiva was conferred by mutations accumulating in the RNA genome. ${ }^{4}$

During October 2007, an epidemic of AHC occurred in Taiwan. Cases were first noted in the northern areas of Taiwan, including Keelung city, and Yunlin county. Within the next few days, the disease spread to Sijhih, a city in Taipei county south of Keelung, and Taipei city. As monitored by the Center of Disease Control in Taiwan, the areas involved in the epidemic of AHC 2007 included Keelung city, Taipei city and county, Yunlin county, and Chiayi county. More than 11000 cases were officially reported to the public health authorities in the first 2 weeks of October 2007 (CDC Taiwan). ${ }^{5}$ A growing number of patients visited CGHT and CGHS complaining of 'red eye' during this period. They presented with the following conjunctival symptoms, including foreign body sensation, red eye, tearing, eyelid oedema, and subconjunctival haemorrhage (Table 1). Some patients also had systemic symptoms, including headaches and fever, but none of them presented with paralysis of body and face during the course of followups. All patients were diagnosed clinically as cases of acute haemorrhagic conjunctivitis. More than 400 clinically diagnosed cases of acute conjunctivitis alone were noted in CGHS.

To better characterize this outbreak, we collected samples from the conjunctival, throat, and rectal swabs in a group of patients treated at the CGHT and CGHS, and isolated the virus through the MRC- 5 cell line.

Subsequently, we identify the aetiological agent through immunocytochemical studies with IFA, and molecular studies with a one-step real-time RT-PCR assay based on the TaqMan technology, which allows for the rapid and sensitive detection of the specific genre of the CA- $24 \mathrm{v} .{ }^{6}$

\section{Materials and methods}

\section{Clinical samples and virus isolation}

Patients were selected randomly from those who visited the hospitals with symptoms of AHC during the period of September and October 2007 at the CGHT and CGHS. Clinical specimens were obtained from six patients (four from CGHS and two from CGH), and a total of seven specimens ( one throat, and one rectal, and five eye swabs) were collected. (Table 1) All applicable institutional and governmental regulations concerning the ethical use of human volunteers were followed during this study.

A total of seven clinical samples (one throat, and one rectal, and five eye swabs) were treated according to the method, described earlier, used for conventional virus isolation. $^{2}$ A confluent monolayer of MRC-5 cells (BCRC number: 60023) maintained in the minimum essential medium (GIBCO, Invitrogen, CA, USA) with $2 \%$ foetal bovine serum (GIBCO, Invitrogen) under $36^{\circ} \mathrm{C} / \mathrm{CO}_{2}(5 \%)$ condition was inoculated with the above treated specimens. The inoculated cells were checked daily for the presence of cytopathic effect (CPE). When CPE was observed, the cells were scraped, smeared onto a glass slide, air dried, and fixed in acetone for $15 \mathrm{~min}$.

\section{Indirect immunofluorescence assay (IFA)}

The slides with the virus-infected cells were tested with Light Diagnostics ${ }^{\mathrm{TM}}$ coxsackie A24 virus monoclonal antibody (Millipore Ltd., UK) and incubated at $37^{\circ} \mathrm{C}$ in a humid chamber for $30 \mathrm{~min}$. After incubation, the slides were washed twice in PBS, and rinsed with distilled water. The slides were further tested with goat antimouse IgG conjugated with fluorescein isothiocyanate, incubated in a humid chamber at $37^{\circ} \mathrm{C}$ for $30 \mathrm{~min}$, washed twice in PBS, rinsed with distilled water, and air dried. Subsequently, the slides were mounted in a mounting fluid (Imagen, DakoCytomation, Denmark) and observed with a fluorescence microscope. Normal mouse antibody was used as a negative control. Evans Blue exhibiting a dull red background hue was used as a counter stain for both infected and non-infected cells. Cells exhibiting an apple-green fluorescence were considered positive for CA-24v.

\section{Viral RNA isolation and real-time RT-PCR assay}

The processing of viral RNA isolation and real-time RT-PCR were performed as described earlier. ${ }^{6}$ In brief, the viral RNA was isolated from $140 \mu \mathrm{l}$ of cell culture supernatants with the QIAamp Viral RNA minikit (QIAGEN, International Inc.) and eluted in $60 \mu \mathrm{l}$ of the 
Table 1 Characteristics including signs and symptoms of the six patients with AHC

\begin{tabular}{|c|c|c|c|c|c|c|}
\hline & Patient 1 & Patient 2 & Patient 3 & Patient 4 & Patient 5 & Patient 6 \\
\hline Age & 10 years & 35 years & 57 years & 2 months & 2 years & 8 years \\
\hline Sex & M & $\mathrm{F}$ & $\mathrm{F}$ & $\mathrm{F}$ & $\mathrm{F}$ & $\mathrm{M}$ \\
\hline Swab & Eye & Eye & Eye & Throat and rectal & Eye & Eye \\
\hline \multicolumn{7}{|l|}{ Signs and symptoms } \\
\hline $\mathrm{CPH}$ & + & + & + & - & - & - \\
\hline FBS & + & + & + & - & - & + \\
\hline Tearing & + & + & + & - & - & - \\
\hline Redness & + & + & + & + & + & + \\
\hline Photophobia & - & - & - & - & - & - \\
\hline Keratitis & - & - & - & - & - & - \\
\hline Pseudomembranes & - & - & - & - & - & - \\
\hline Follicle reactions & + & + & + & - & - & - \\
\hline Chemosis & - & - & - & - & - & - \\
\hline Eyelid oedema & - & - & - & - & - & - \\
\hline Discharge & + & + & + & + & + & + \\
\hline Fever & - & - & - & + & - & + \\
\hline Headache & - & - & - & - & - & - \\
\hline
\end{tabular}

$\mathrm{AHC}=$ Acute haemorrhagic conjunctivitis; $\mathrm{CPH}=$ conjunctival petechial hemorrhage; $\mathrm{FBS}=$ foreign body sensation.

buffer AVE. Four microlitres of template RNA were added to 201 of a mix, including the TaqMan one-step RT-PCR Master Mix reagents (Applied Biosystems, NJ, USA), $900 \mathrm{nM}$ of the forward (5'-CCAACCACGGAGC AGGTGA-3') and reverse (5'-GAAACACGGACACC CAAAGTAGT- $\left.3^{\prime}\right)$ primers, and $200 \mathrm{nM}$ of the probe (5'-CAACCCAGCAACTAGCCTGTCGTAACGC-3').

The cycling profile performed using the 7300 real-time RT-PCR System (Applied Biosystems) involved reverse transcription at $48^{\circ} \mathrm{C}$ for $30 \mathrm{~min}$ and initial denaturation at $95^{\circ} \mathrm{C}$ for $10 \mathrm{~min}$ followed by 40 cycles for $15 \mathrm{~s}$ at $95^{\circ} \mathrm{C}$ and for $1 \mathrm{~min}$ at $60^{\circ} \mathrm{C}$. Fluorescence data were collected during each annealing-extension step and analysed using software 7300 system version 1.6.3 (Applied Biosystems). The sensitivity of the test was then evaluated using 10-fold dilution series of titrated CV-A24v strains. Furthermore, the dilution series of each sample were tested four times to determine the lowest concentration that can be reproducibly detected.

\section{Results}

The epidemics began in the first week of September. It spread explosively from the northern part of Taiwan, with the number of patients peaking in the middle of October and subsiding during November (Figure 1).

The patients included in the study ranged in age from 2 months to 57 years. The symptoms lasted 5-14 days. As little information was available on the age distribution of these patients, this part of the data could not be analysed in as much detail. The school-age group occupied the largest portion of the patients, and there seems to be no significant difference between genders.
All seven specimens of swabs tested in this study were inoculated onto the MRC-5 cell lines (Table 1). After 2-3 days of the inoculation, the MRC-5 cells started to express CPE. When the CPE increased more than 50\%, IFA assay was performed to examine the presence of coxackievirus A24. Enterovirus-specific cytopathic effects were observed in all seven specimens, which were positive for CA-24v after the IFA study (Figure 2). Real-time RT-PCR assay revealed that the CA- $24 \mathrm{v}$ isolates that were collected belonged to a distinct lineage within genotype III similar to the earlier Asian isolates. ${ }^{7}$ The cell culture supernatants from all the swab inoculations were tested positive for the CA-24v by realtime RT-PCR assay. The range of $C_{t}$ values observed in the undiluted cell culture supernatants is from 29 to 32 . Each of the samples was then diluted 10-, 100-, and 1000-fold, and real-time RT-PCR assay was repeated for each of the diluted samples. The $C_{t}$ values were found to increase linearly with the dilutions in all the samples, indicating the sensitivity of the assay $\left(R^{2}=0.996\right.$; Figure 3). As the IFA is a well-recognized method in enterovirus typing, the identification of CA-24v by IFA combined with real-time RT-PCR confirmation should be legitimate and convincing.,

\section{Discussion}

The symptoms of AHC were first noted in 1969 in Ghana, West Africa, from where the disease spread as a pandemic to the rest of the world. ${ }^{10-12}$ The disease was noted to be unusual because of its rapid spread, and fast onset, and recovery of clinical symptoms. Although the Ghana report originally suggested that AHC was caused 
by the adenovirus, later studies have identified the enterovirus 70 (EV-70) as the aetiological agent. ${ }^{13}$ Although the first outbreak caused by EV-70 appeared first in West Africa, CA-24v emerged in Singapore for the first time in 1970 and was restricted to Southeast Asian countries and India from 1970 to 1985, until the third outbreak in Singapore in $1985^{14}$ from where it spread rapidly to Malaysia, ${ }^{15}$ Japan, Thailand, ${ }^{16}$ South Korea, ${ }^{17,18}$ and Taiwan, ${ }^{19,20}$ and eastward to the Americas. ${ }^{21,22}$

In Taiwan, the first major outbreak of AHC occurred in 1971 with EV-70 identified as the causative agent. Between the years 1971 and 1985, multiple endemic outbreaks caused by EV-70 confirmed by virus isolation and identification, were reported. Thereafter, however, four major outbreaks of AHC reported in 1985-1986, 1988-1989, and in 1990-1994 were all caused by different

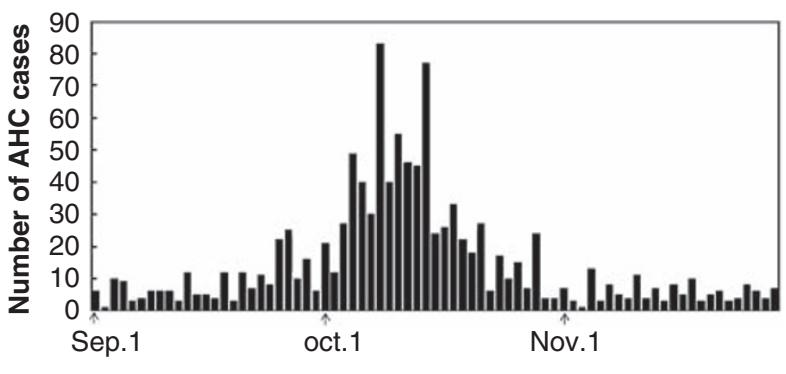

Date (from Sep. 1 to Nov. 30, 2007)

Figure 1 Number of patients with AHC by the day in CGHT and CGHS from September to November 2007. In 2007, the AHC epidemics in Taiwan begins in the first week of the September, peaks in the middle of October, and subsides afterwards. The $X$ axis indicates time points. The $Y$ axis indicates the number of patients diagnosed with AHC during each specific time point. strains of CA-24v, rather than EV-70.7,19,20,23,24 This is supported by the serological evidence, from the serum of the Taiwanese population before the 1985-1986 outbreak, which indicates high titres of antibodies against EV-70 and very low titres of antibodies against CA-24v. ${ }^{19}$ During September and October 2007, a major outbreak of AHC occurred again in Taiwan, with an estimate of more than 11000 people affected. Phylogenic studies revealed the causative agent being CA- $24 \mathrm{v}$, genotype III; which is the same genotype as the virus strain isolated from the AHC outbreaks in Taiwan, Japan, China, Hong-Kong, and Singapore between 1985 and 2003. ${ }^{15}$

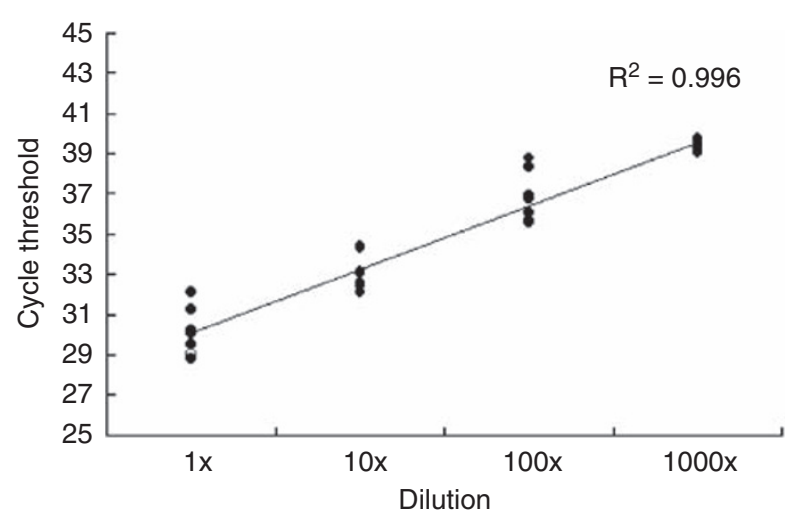

Figure 3 Molecular identification of CA-24v by Real-time RT-PCR. The range of $C_{t}$ values varied from 29 to 32 in undiluted cell culture supernatants from the samples. The sensitivity was assessed by repeating the assay in 10, 100 and 1000 fold dilution series of viral RNA of CA-24v strains from each sample. The increased cycle threshold associated with the increased dilution showed good sensitivity of the RT-PCR assay.
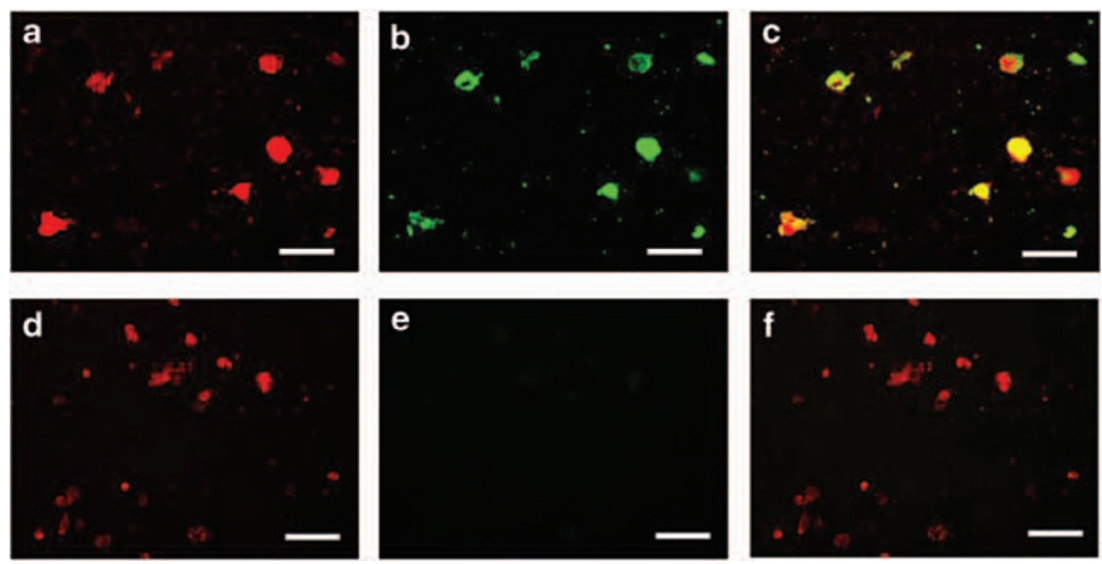

Figure 2 Indirect immunofluorescence assay showing CA-24v cytopathic effect on the MRC-5 cells. (a-c) Staining results using Light Diagnostics ${ }^{\mathrm{TM}}$ Coxsackie A24 Virus Monoclonal Antibody are shown. (a) Dull red staining with the Evans Blue counter stain in infected MRC-5 cells. (b) Apple-green cell fluorescence in MRC-5 cells infected with CA-24v when illuminated with ultraviolet light allowing visualization of the complex by microscopy. Figure $2 c$ is a merge of the microscopy images of panels a and b). (d and e) Only background dull red stain without apple-green cell fluorescence when infected MRC-5 cells are stained with normal mouse antibody. A merge of the microscopy images of panels $d$ and e is shown (f). Scale bar: $50 \mu \mathrm{m}$. 
In our study, besides the five conjunctival swabs, which were positive for the CA-24v virus isolation, specimens from the throat and rectal swabs from the same AHC patient also cultured positive for the CA-24v virus. The results were significant for two reasons. First, besides the conjunctival swabs, the throat and rectal swabs may also provide a good source for viral isolation. Second, the results suggested that the CA-24v can also be transmitted by ways other than the earlier suggested close person-to-person contact. Respiratory droplet may also be a possible route of transmission. Therefore, AHC may not only be spread through conjunctival secretions, but also through the respiratory and fecal-oral route. The multiple ways of transmission may account for the rapid and explosive spread of CA-24v within the communities and to the neighbouring countries.

This study also reminds us of the importance of virus isolation in any outbreak for subsequent virus identification. An earlier study has described a rapid method of identifying the virus through the use of real-time RT-PCR directly from the collected samples. However, it also indicated the possibility of getting negative results from the assay secondary to the limitation of the technique and sampling methods. ${ }^{6}$ Our experiment has shown that when the causative agent is unknown initially, the procedure of virus isolation is important for the following reasons: first, as the samples collected are always contaminated, isolating, and preserving, the virus strain provide a cleaner specimen for further detailed phylogenic studies; second, the virus preserved may function as a backup specimen in case the real-time RT-PCR performed directly from the sample fails to give results. Virus isolation in any outbreak, although time consuming, is a necessary procedure for the later identification of the virus as the procedure allows the preservation of the virus strain.

This report is the first to identify CA- $24 \mathrm{v}$ as the aetiological agent that is responsible for the 2007 September-November AHC outbreak in Taiwan. Whether this virus is the same strain as the virus strains from the last outbreak in Taiwan remains to be elucidated. Further phylogenic analysis is currently underway to classify the virus strain into clusters. The virus isolates obtained in the 1985/1986, 1988/1989, and 1990 outbreaks were classified into genotype III clusters 1,5 , and 6 , respectively. ${ }^{19,20}$ Comparison of the nucleoside sequences of the virus isolates of the outbreaks with those of the earlier outbreaks will help to identify the phylogenic relationships among the different strains. The results will provide insights into the origin of this outbreak as to whether this outbreak was caused by the resurgence of the same virus strains from the earlier outbreaks in Taiwan or by different viruses introduced from nearby countries.

\section{Acknowledgements}

This study was supported in part by research grants from Cathay General Hospital, National Central University Funding (96CGH-NCU-A1).

\section{References}

1 Kono R, Sasagawa A, Ishii K, Sugiura S, Ochi M. Pandemic of new type of conjunctivitis. Lancet 1972; 1: 1191-1194.

2 Wright PW, Strauss GH, Langford MP. Acute hemorrhagic conjunctivitis. Am Fam Physician 1992; 45: 173-178.

3 Yin-Murphy M, Goh KT, Phoon MC, Yao J, Baharuddin I. A recent epidemic of acute hemorrhagic conjunctivitis. Am J Ophthalmol 1993; 116: 212-217.

4 Holland J, Spindler K, Horodyski F, Grabau E, Nichol S, VandePol S. Rapid evolution of RNA genomes. Science 1982; 215: 1577-1585.

5 Chen WL. Acute hemorrhagic conjunctivitis - a brief review. Epidemiol Bull (Taiwan) 2008; 24: 289-302.

6 Leveque N, Lahlou Amine I, Tcheng R, Falcon D, Rivat N, Dussart $\mathrm{P}$ et al. Rapid diagnosis of acute hemorrhagic conjunctivitis due to coxsackievirus A24 variant by realtime one-step RT-PCR. J Virol Methods 2007; 142: 89-94.

7 Chou MY, Malison MD. Outbreak of acute hemorrhagic conjunctivitis due to coxsackie A24 variant-Taiwan. Am J Epidemiol 1988; 127: 795-800.

8 Bastis D, Simonet S, Patterson MA, Neill S. Identification of enteroviruses by indirect immunofluorescence using monoclonal antibodies. Clin Diagn Virol 1995; 3: 83-93.

9 French ML, Schmidt NJ, Emmons RW, Lennette EH. Immunofluorescence staining of group B coxsackieviruses. Appl Microbiol 1972; 23: 54-61.

10 Chatterjee S, Quarcoopome CO, Apenteng A. An epidemic of acute conjunctivitis in Ghana. Ghana Med J 1970; 9: 9-11.

11 Chatterjee S, Quarcoopome CO, Apenteng A. Unusual type of epidemic conjunctivitis in Ghana. Br J Ophthalmol 1970; 54: 628-630.

12 Kono R. Apollo 11 disease or acute hemorrhagic conjunctivitis: a pandemic of a new enterovirus infection of the eyes. Am J Epidemiol 1975; 101: 383-390.

13 Leveque N, Amine IL, Cartet G, Hammani AB, Khazraji YC, Lina B et al. Two outbreaks of acute hemorrhagic conjunctivitis in Africa due to genotype III coxsackievirus A24 variant. Eur J Clin Microbiol Infect Dis 2007; 26: 199-202.

14 Lim KH, Yin-Murphy M. An epidemic of conjunctivitis in Singapore in 1970. Singapore Med J 1971; 12: 247-249.

15 Ghazali O, Chua KB, Ng KP, Hooi PS, Pallansch MA, Oberste MS et al. An outbreak of acute haemorrhagic conjunctivitis in Melaka, Malaysia. Singapore Med J 2003; 44: 511-516.

16 Dussart P, Cartet G, Huguet P, Leveque N, Hajjar C, Morvan $\mathrm{J}$ et al. Outbreak of acute hemorrhagic conjunctivitis in French Guiana and West Indies caused by coxsackievirus A24 variant: phylogenetic analysis reveals Asian import. J Med Virol 2005; 75: 559-565.

17 Oh MD, Park S, Choi Y, Kim H, Lee K, Park W et al. Acute hemorrhagic conjunctivitis caused by coxsackievirus A24 variant, South Korea, 2002. Emerg Infect Dis 2003; 9: 1010-1012.

18 Park K, Lee K, Lee J, Yeo S, Lee S, Cheon DS et al. Acute hemorrhagic conjunctivitis epidemic caused by 
coxsackievirus A24 variants in Korea during 2002-2003. J Med Virol 2006; 78: 91-97.

19 Lin KH, Wang HL, Sheu MM, Huang WL, Chen CW, Yang $\mathrm{CS}$ et al. Molecular epidemiology of a variant of coxsackievirus A24 in Taiwan: two epidemics caused by phylogenetically distinct viruses from 1985 to 1989. J Clin Microbiol 1993; 31: 1160-1166.

20 Lin KH, Sheu MM, Huang L, Chen CW, Wen KH, Hwang $\mathrm{HZ}$ et al. Seroepidemiological study of coxsackievirus type A24 variant (CA24v) in Taiwan. Gaoxiong Yi Xue Ke Xue Za Zhi 1994; 10: 606-612.

21 Kuritsky JN, Weaver JH, Bernard KW, Mokhbat JE, Hatch $\mathrm{MH}$, Osterholm MT et al. An outbreak of acute hemorrhagic conjunctivitis in central Minnesota. Am J Ophthalmol 1983; 96: 449-452.

22 Asbell PA, de la Pena W, Harms D, Hatch M, Kaufman HE. Acute hemorrhagic conjunctivitis in Central America: first enterovirus epidemic in the western hemisphere. Ann Ophthalmol 1985; 17: 205-208, 210

23 Lin KH, Chern CL, Chu PY, Chang CH, Wang HL, Sheu MM et al. Genetic analysis of recent Taiwanese isolates of a variant of coxsackievirus A24. J Med Virol 2001; 64: 269-274.

24 Lin KH, Takeda N, Miyamura K, Yamazaki S, Chen CW. The nucleotide sequence of $3 \mathrm{C}$ proteinase region of the coxsackievirus A24 variant: comparison of the isolates in Taiwan in 1985-1988. Virus Genes 1991; 5: 121-131. 